\title{
Requests from primary care for chest X-ray and CA125 measurements during the COVID-19 emergency: An observational study
}

\author{
Authors: S Michael Crawford, ${ }^{A}$ Colin Evans, ${ }^{B}$ Heather Edwards ${ }^{C}$ and Anna Zoltowski ${ }^{C}$
}

During the first 3 months of 2020, as the COVID-19 pandemic developed, it was noticed that requests from primary care for investigations were decreasing, including those that form part of the diagnostic process for cancers. We therefore obtained data on the requests from primary care for chest X-rays (CXRs) and CA125 measurement our hospital received in the first half of 2020 and compared them with 2019. The number of CXRs declined by $93 \%$ in April 2020 compared with 2019, with the decline being greater for patient living in outlying areas. Requests from the emergency department also declined. Requests for CA125 measurement similarly fell by $77 \%$ from all areas. The requests increased in June, CA125 more than CXR. If this phenomenon is widespread it may have an impact on diagnosis of major conditions, particularly cancers and tuberculosis.

KEYWORDS: primary care, diagnostic tests, chest X-ray, CA125, COVID-19

DOI: $10.7861 /$ clinmed.2020-0638

\section{Introduction}

During the COVID-19 pandemic of 2020 the practice of medicine in the UK has undergone profound changes. 'The first cases of the disease in the UK at the end of January were visitors from China but over the next 2 months it became apparent that there was transmission taking place within the country and in late March lockdown procedures were enacted by the government to minimise this. The process resulted in changing emphasis within the NHS so that resources were devoted to the anticipated rising incidence of COVID-19 and the need to manage the consequences of that at the cost of diminishing other services including access to diagnostic services for cancer; urgent referrals decreased by $76 \%$ averaged over the hospitals studied. ${ }^{2}$ Other major conditions showed similar trends. Primary care practice reflected what was happening in the

Authors: ${ }^{A}$ clinical lead for research, Airedale NHS Foundation Trust, Steeton, UK; ${ }^{B}$ consultant biochemist, Airedale NHS Foundation Trust, Steeton, UK; ' Consultant radiologist, Airedale NHS Foundation Trust, Steeton, UK

\section{Key points}

> Routine hospital functions have been disrupted during the COVID-19 pandemic.

> We sought to identify associated changes in specific diagnostic tests requested from primary care.

> Both chest X-ray and CA125 requests decreased sharply as the outbreak developed with some subsequent recovery in CA125 requests but less so with CXRs.

> This is relevant to understanding future changes in cancer and other referrals in the coming months.

community and it was noted at Airedale General Hospital that the number of requests from primary care for chest radiography had decreased. It was also noted that laboratory requests from primary care were decreasing.

In relation to the diagnosis of cancer, some investigations are intended to be conducted in primary care in order to instruct the decision to refer a patient to the diagnostic service for possible cancer. Among the tests GPs are advised to undertake for this purpose are chest $X$-rays (CXR) for thoracic tumours and CA125 when carcinoma of the ovary is possible. ${ }^{3}$ Delay in, or omission of, performance of these tests may therefore delay entry of the patient into the cancer diagnosis pathway. There was no restriction on the part of the biochemistry laboratory in accepting samples. The radiology department had a policy during the month of May 2020 of asking GPs to discuss requests with a radiologist. We explored this by obtaining the numbers of requests from primary care for a CXR and requests for CA125 measurement for detection of possible ovarian cancer. The purpose was to compare activity in 2020 with the corresponding month in 2019.

\section{Methods}

We analysed data from all adult patients referred from primary care for CXR at Airedale General Hospital and from CA125 blood tests in in the Airedale laboratory from GPs whose patients are routinely referred to that hospital. The only information obtained about each patient was the postcode. We excluded requests from outpatient clinics, inpatient wards and the oncology treatment unit. We compared the number of requests from surrounding areas (Craven District, Wharfe Valley, Bingley and Bradford outskirts) with the town of Keighley (postal districts BD20-BD22) on the edge of which Airedale General Hospital is located. 
Table 1. Requests for chest $X$-ray from primary care

$\begin{array}{lllllll} & \text { Jan } & \text { Feb } & \text { Mar } & \text { Apr } & \text { May } & \text { Jun } \\ \mathbf{2 0 1 9} & 899 & 877 & 827 & 814 & 957 & 655 \\ \mathbf{2 0 2 0} & 960 & 761 & 612 & 58 & 78 & 217 \\ \text { Ratio } & 1.07 & 0.87 & 0.74 & 0.07 & 0.08 & 0.33\end{array}$

For comparison, the number of patients having CXRs requested from the emergency department (ED) is given.

As this was a report of overall activity in the services and individual patient details were not scrutinised this study has not been subject to ethical review.

\section{Results}

The number of referrals and CA125 requests during January and February was similar between 2019 and 2020. There was a modest reduction in March and a very marked reduction, especially in the CXR, in April and May.

Table 1 shows radiology requests and Table 2 CA125 requests. Chest radiographs requested from primary care were $93 \%$ fewer in April 2020 compared with 2019. Table 3 shows the comparator data from the ED.

Requests for CA125 were $77 \%$ fewer in April 2020 compared with the previous year. The figure for May 2020 was improved slightly but significantly to $62 \%$ fewer than 2019 (odds ratio for May compared with April 1.64, 95\% confidence interval [CI]1.16-2.32) and more so in June (14\% fewer, odds ratio for June compared with May 2.55, 95\% CI 1.91-3.39).

In regard to where the patients lived for whom radiographs were requested, the number of requests from the areas further from the hospital fell more sharply than those from Keighley. This difference did not reach significance in May or June (Table 4). No comparable differences in residential location were seen in respect of CA125 (data not presented).

For both CA125 and CXR requests, we looked for differences in socioeconomic status (Index of Multiple Deprivation) associated with the immediate locality of residence (lower super output area) associated with the postcode and found no pattern.

\section{Discussion}

There are differences in the way tests for patients in whom cancer is recognised as a potential diagnosis, as advised by the National Institute for Health and Care Excellence, are initiated from primary care. $^{3}$ For example, direct-access endoscopy was suspended early in the peak of the COVID-19 pandemic and as reopening takes place it is recommended that referrals should be subject to vetting before the patient attends. ${ }^{4}$

In contrast, the tests whose usage we have studied are conducted as part of routine primary care practice, usually using the facilities of the local general hospital trust. The sole

Table 2. Requests for serum CA125 from primary care

$\begin{array}{lllllll} & \text { Jan } & \text { Feb } & \text { Mar } & \text { Apr } & \text { May } & \text { Jun } \\ \mathbf{2 0 1 9} & 300 & 304 & 282 & 266 & 302 & 244 \\ 2020 & 247 & 250 & 195 & 63 & 117 & 211 \\ \text { Ratio } & 0.82 & 0.82 & 0.69 & 0.23 & 0.38 & 0.86\end{array}$

\begin{tabular}{|c|c|c|c|c|c|c|}
\hline & Jan & Feb & Mar & Apr & May & Jun \\
\hline 2019 & 935 & 844 & 880 & 842 & 890 & 891 \\
\hline 2020 & 959 & 812 & 725 & 738 & 757 & 726 \\
\hline Ratio & 1.03 & 0.96 & 0.82 & 0.88 & 0.85 & 0.82 \\
\hline
\end{tabular}

indication for measuring serum CA125 in primary care is to address the possibility of ovarian cancer. It is an effective triage test. ${ }^{5}$ There are many reasons for requesting CXR; diagnosis of serious conditions such as thoracic malignancy or pulmonary tuberculosis is one important outcome even if these were not under consideration when the request was made and are raised by the reporting clinician. The sharp reduction in these investigations during the first months of the lockdown in response to the COVID-19 pandemic is therefore concerning. The fact that the decline in CA125 requests did not vary with distance from the hospital does not mean that the underlying rates of referral do not differ with these factors, as is well established. ${ }^{6,7}$

Reasons for these reductions include patients feeling it inappropriate to turn to any medical facility for minor symptoms during the pandemic. This may be reinforced if a telephone call is answered with a recorded message, as in this local example: 'If you have a new cough... do not contact your GP, a hospital or a pharmacy.' The difference between a new cough as a symptom of COVID-19 and a persistent cough as a symptom of lung cancer or tuberculosis may not be appreciated by an anxious patient.

It is reported anecdotally that many patients believed that GP practices were not open. They may also have been reluctant to attend the hospital for a test because of a perception that many patients carrying COVID-19 infection would be present there. Radiology department staff members report that patients state this in conversations about arranging appointments. Seemingly small barriers to accessing the service, such as distance from the radiology service are apparently an influence, as seen in Table 4. GPs are reported to have advised patients to go to the ED rather than the GP practice if they had respiratory symptoms. However, our data (Table 3 ) show a slight decline in the number of CXRs requested from there. This level of referral has persisted into June.

Primary care professionals may seek to defend the general hospital from work considered not as urgent as that related to the pandemic. Such defensiveness has been approved in a senior hospital physician's personal opinion with regard to access to cancer diagnostic services before the pandemic. ${ }^{8}$ This process may be compounded if the provider requires a telephone call for justification of an investigation before the test could be enacted. This was a temporary requirement at Airedale General Hospital during May 2020. Sometimes this resulted in CT scan being performed rather than CXR, a potentially favourable change. A significant proportion of CXRs from primary care are for follow up. These were not done during the period studied, with the interval being advised to plan such an investigation being increased from 4-6 weeks to 12 weeks.

A further possibility is that, compared with a face-to-face consultation, in a telephone or videoconferencing conversation the GP may not as effectively elicit the symptomatic clues which prompt the investigation or indeed referral. The potential for these 
Table 4. Comparison of reduction in chest $\mathrm{X}$-ray requests by area of residence

\begin{tabular}{lllllllll}
\hline & Mar 2019 & Mar 2020 & Apr 2019 & Apr 2020 & May 2019 & May 2020 & Jun 2019 & Jun 2020 \\
$\begin{array}{l}\text { Keighley postcodes } \\
\text { (BD20-22) }\end{array}$ & 414 & 325 & 324 & 37 & 486 & 37 & 312 & 120 \\
$\begin{array}{l}\text { Other surrounding area } \\
\text { Odds ratio (95\% }\end{array}$ & 490 & 287 & 490 & 21 & 471 & 41 & 655 & 217 \\
\begin{tabular}{l} 
confidence interval) \\
\hline
\end{tabular} & 0.75 & & 0.38 & & 0.81 & & 0.74 & $(0.54-1.00)$ \\
\hline
\end{tabular}

Odds ratio $<1$ means referrals from other postcodes decreased more than from BD20-22.

changes to be part of normal primary care function will therefore need to be very carefully evaluated. ${ }^{1,9}$

The reversion of the CA125 measurements to rates approaching those of the previous year suggests that the issue of potential ovarian cancer is being considered by GPs and that patients are again presenting with relevant symptoms. The requests for chest radiography are not increasing at the same rate, which raises some concerns; the geographical variation would suggest that travel to the hospital is a consideration for patients.

The number of ' 2 week wait' referrals to Airedale General Hospital for investigation of potential cancer fell by $60 \%$ from 554 in April 2019 to 220 in April 2020.10 This forms part of a general pattern of reduced activity in primary care, which would lead to delayed cancer diagnosis even before the delays in workup of patients that have accessed the specialist services. In discussing the effect of the pandemic on cancer diagnostic processes and the potential effect on outcomes, Hamilton describes services as being in disarray.11

The observations we report pertain to the actions of GPs using the services of one NHS acute hospital. The reduction of activity they represent may differ from the experience in other districts. However, since in the UK referrals from primary care have reduced widely during the first half of 2020 some similar findings are likely around the country.

As the management of the 2020 pandemic is developed, changes in activity will need to be identified and taken into account. It is not possible in a retrospective audit such as this study to identify the contributory factors to the reduction in diagnostic tests but to conduct such research would be valuable. Its findings would instruct planning for future major outbreaks of infectious disease. Continued monitoring of the way such facilities are used will be necessary as usual activity is resumed within the NHS.

\section{Acknowledgements}

We are grateful to Anna Curini and Gillian Day for supplying data and to Girish Raghunathan for his advice.

\section{References}

1 Khan N, Jones D, Grice A. A brave new world: the new normal for general practice after the COVID-19 pandemic. BJGP Open 2020;4:bjgpopen20X101103.

2 Lai AG, Pasea L, Banerjee A. Estimating excess mortality in people with cancer and multimorbidity in the COVID-19 emergency. medRxiv 2020;2020.05.27.20083287.

3 National Institute of Health and Care Excellence. Suspected cancer: recognition and referral. NICE guideline [NG12]. NICE, 2015 (updated 2020). Available from www.nice.org.uk/guidance/ng12 [Accessed 8 June 2020].

4 Rees C], East JE, Oppong $\mathrm{K}$ et al. Restarting gastrointestinal endoscopy in the deceleration and early recovery phases of COVID-19 pandemic: Guidance from the British Society of Gastroenterology. Clin Med 2020;20:252-8.

5 Crawford SM, Evans C. Outcome of elevated CA125 values from primary care following implementation of ovarian cancer guidelines. Fam Pract 2018;35:199-202.

6 McCutchan G, Wood F, Smits S et al. Barriers to cancer symptom presentation among people from low socioeconomic groups: a qualitative study. BMC Public Health 2016;16:1052.

7 Peake MD. Deprivation, distance and death in lung cancer. Thorax 2015;70:108-9.

8 Oliver D. Don't blame GPs for late cancer diagnoses. BM] 2019;366:14625.

9 Mathew R. Patients want to choose how they see a GP. BMJ 2020;370:m2654.

10 NHS England. Cancer waiting times. www.england.nhs.uk/statistics/ statistical-work-areas/cancer-waiting-times/ [Accessed 13 ]une 2020].

11 Hamilton W. Cancer diagnostic delay in the COVID-19 era: what happens next? Lancet Oncol 2020;21:1000-2.

Address for correspondence: Dr S Michael Crawford, Airedale NHS Foundation Trust, Skipton Road, Steeton, Keighley, West Yorkshire BD20 6TD, UK.

Email: s.m.crawford@doctors.org.uk 